\title{
Transient Expression Assay in Strawberry Fruits
}

Mengting Pi, Qi Gao and Chunying Kang*

Key Laboratory of Horticultural Plant Biology (Ministry of Education), College of Horticulture and Forestry Sciences, Huazhong Agricultural University, Wuhan, 430070, China

*For correspondence: ckang@mail.hzau.edu.cn

[Abstract] Strawberry, including the woodland strawberry Fragaria vesca $(2 x)$ and the cultivated strawberry (Fragaria $\times$ ananassa, $8 x$ ), has emerged as a model system for studying fruit development and ripening. Transient expression provides a quick assay for gene functions or gene interactions. In strawberry, virus-induced gene silencing (VIGS) and Agrobacterium tumefaciens-mediated transformation in fruit have been widely used as the transient expression approaches. Unlike VIGS, the latter one can be utilized not only for gene knock-down, but also for overexpression and knock-out. Here, we show the procedures of transiently expressing the 35S::FveMYB10 construct into fruit of the white-fruited $F$. vesca accession Yellow Wonder. As a master regulator of anthocyanin production, overexpressing FveMYB10 will cause fruit coloration, which was observed at one week post infiltration. We also exhibit the previous results of knocking down Reduced Anthocyanin in Petioles (RAP), encoding an anthocyanin transporter, by RNAi in fruit of the strawberry cultivar 'Sweet Charlie'. Overall, Agrobacterium-mediated transient transformation in strawberry fruit is a quick and versatile approach for studying gene functions in fruit ripening.

Keywords: Transient expression, FveMYB10, Overexpression, RAP, RNAi, Agrobacterium, Strawberry

[Background] Cultivated strawberry (Fragaria $\times$ ananassa, $8 x$ ) is an economically important fruit crop grown worldwide with lovely appearance and rich nutrition. The wild diploid strawberry Fragaria vesca has emerged as a model plant for cultivated strawberry as well as other fleshy fruit species. Moreover, strawberry is a typical non-climacteric fruit, studies on the ripening of which lack a nice model system, like tomato as a model of the climacteric fruit. Therefore, strawberry is also frequently used for studying fruit ripening.

Stable transformation is doable in both woodland and cultivated strawberry; however, the entire process is labor-intensive and time-consuming. In contrast, the transient expression assay in fruit is much faster and more efficient. There are two transient gene expression approaches in strawberry, namely Virus-Induced Gene Silencing (VIGS) and Agrobacterium-mediated transformation (Spolaore et al., 2001). Some studies obtained nice results by using VIGS (Jia et al., 2011; Li et al., 2019). However, we utilize the Agrobacterium-mediated transformation more frequently as it can achieve more experimental aims, including gene knock-down (Hoffmann et al., 2006), overexpression (Huang et al., 2018), and knock-out (Tang et al., 2018). 
The F. vesca fruit takes 25-30 days from being pollinated to ripen under our growth conditions. The entire process is divided into seven stages: flower/anthesis, small green, medium green, large green, white, turning, and red. Fruit receptacle (the juicy flesh) at the large green stage, when the firmness starts to decline rapidly (before the achenes turn red for the red strawberry fruit), is suitable for agrobacterium injection. Fruit receptacles at prior stages are recalcitrant to the injection owing to the texture. Thus, Agrobacterium-mediated transformation in strawberry fruit delimits functional studies on genes acting at late developmental stages.

The bright red color of strawberry fruit is caused by the accumulation of anthocyanin compounds, which is a visible phenotype to check. In strawberry, the R2R3-MYB transcription factor FveMYB10 is a master regulator of anthocyanin synthesis (Lin-Wang et al., 2014). The woodland strawberry includes both red-fruited and white-fruited varieties, owing to a natural SNP occurred in FveMYB10; overexpression of FveMYB10 results in accumulation of red pigments in fruit of the white-fruited accession Yellow wonder (YW) (Hawkins et al., 2016). Recently, we identified one anthocyanin transporter encoding gene Reduced Anthocyanin in Petioles (RAP) through chemical mutagenesis, knock-down of which greatly reduced fruit pigmentation (Luo et al., 2018).

In this study, as an example, we describe the procedures of transiently overexpressing FveMYB10 in fruit of YW. Moreover, we exhibit the results of knocking down RAP in fruit of the strawberry cultivar 'Sweet Charlie'.

\section{Materials and Reagents}

1. Tips

2. $\mathrm{Tag}$

3. $1 \mathrm{ml}$ syringe with needle (GEMTIER, catalog number: $0.45 \times 16 \mathrm{RW}$ LB)

4. The white-fruited F. vesca accession Yellow Wonder (YW), and the strawberry cultivar 'Sweet Charlie'

5. Binary vectors: pK7WG2D for overexpression and pK7WIWG2D for RNAi. Both vectors contain a 35S::GFP cassette as a visual reporter

6. Target genes: FveMYB10, FvH4_1g22020/gene31413; RAP, FvH4_1g27460/gene31672

7. Yeast extract (OXOID, catalog number: LP0021)

8. Tryptone (OXOID, catalog number: LP0042)

9. Agar (TSINGKE, catalog number: 1182GR500)

10. MS (PhytoTechnology Laboratories, catalog number: M404-50L)

11. GV3101 Chemically Competent Cell (Shanghai Weidi Biotechnology, catalog number: AC1001)

12. $\mathrm{NaCl}$ (HUSHI, catalog number: 10019318, CAS: 7647-14-5)

13. Sucrose (HUSHI, catalog number: 10021418 , CAS: 57-50-1) 
14. Antibiotics, including Kanamycin (Kan), Gentamicin (Gent), Rifampicin (Rif), and Spectinomycin (Spe) (Biofroxx, catalog numbers: 1162GR005, 1463GR001, R3501, and S8040, respectively)

15. Luria Broth (LB) medium (see Recipes)

16. Injection buffer (see Recipes)

17. Antibiotics (see Recipes)

\section{Equipment}

1. Incubator used for the growth of Agrobacterium at $28{ }^{\circ} \mathrm{C}$ (JINGHONG, catalog number: DNP-9022)

2. Double temperature controlled thermostat (MIULAB, catalog number: BTH-100)

3. Centrifuge (Eppendorf, 5424)

4. Clean workbench (AIRTECH, SW-CJ-1FD)

5. Full temperature shaker (Peiying, catalog number: THZ-C-1)

6. Ultra-low temperature refrigerator (ThermoFisher SCIENTIFIC, Thermo Scientific ${ }^{\mathrm{TM}}$ Forma $^{\mathrm{TM}}$ 88000)

7. Fluorescence dissecting stereomicroscope (Leica, catalog number: M205FA)

8. Plant growth room: $25 \pm 3{ }^{\circ} \mathrm{C}, 16 \mathrm{~h}$ light/ $8 \mathrm{~h}$ dark, light intensity at $100 \mu \mathrm{mol} \mathrm{m} \mathrm{sec}^{-1}$

\section{Procedure}

1. Grow strawberry plants in the growth room for 3 to 4 months until the flowers open.

2. Pollinate the flowers manually; wait until the fruits develop to the large green stage, at about 15-20 days post pollination when the receptacle starts to turn soft.

3. Transform the $35 \mathrm{~S}::$ FveMYB10 construct (binary vector pK7WG2D) into the Agrobacterium tumefaciens strain GV3101, or use the Agrobacterium stock stored in the ultra-low temperature refrigerator. Pick a single positive colony and put into $2 \mathrm{ml}$ of the liquid LB medium with the appropriate selective antibiotics, in this case, Gent + Rif + Spec.

4. Shake the culture overnight $(\sim 12-16 \mathrm{~h})$ at $28^{\circ} \mathrm{C}$ at the rate of $220 \mathrm{rpm}$.

5. (Optional) Amplify the fragments in the vector by regular PCR to make sure that the culture is correct.

6. Spin down the culture at 2,500 $\times g$ for $5 \mathrm{~min}$, discard the supernatant. Suspend the culture with the injection buffer (MS salt $+2 \%$ sucrose) to reach a final $\mathrm{OD}_{600}$ of 0.8 .

Note: One milliliter cell suspension is enough for injecting 5-8 fruits. Two milliliters of culture usually makes $4 \mathrm{ml}$ of cell suspension. The volume of the Agrobacterium culture could be increased if more injections are required.

7. Use a $1 \mathrm{ml}$ hypodermic syringe to do the injection immediately. Suck up the solution into the syringe, insert the needle tip into the center of the fruit from the apex, and gently press the 
syringe to release the solution. For a big fruit, such as that of the cultivated strawberry, more injections in other parts of the fruit are sometimes required to make the entire fruit soaked with the solution (Video 1).

Note: Inject at least 10 fruits for each construct. Use fruits infiltrated only with the injection buffer as the negative control.

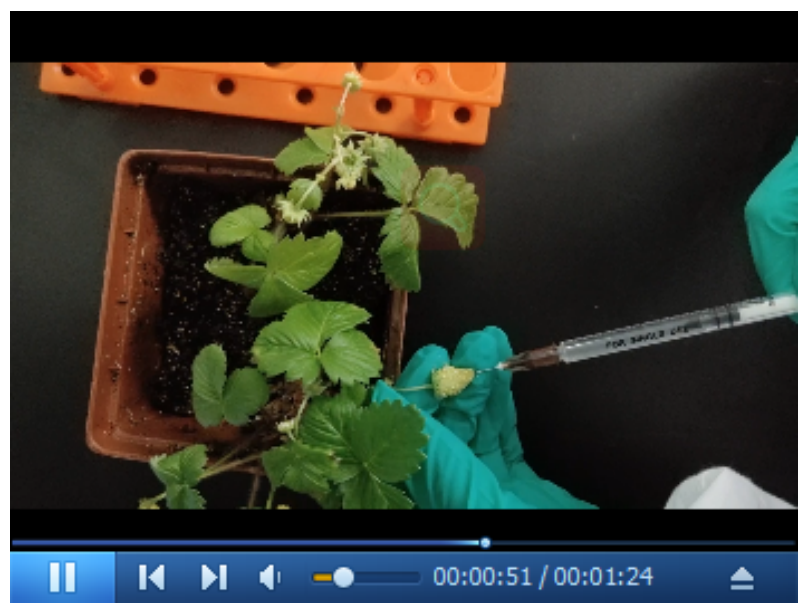

Video 1. Procedure for the fruit injection as stated in Step 7

8. Write down the construct name and the date on the tag, and tie the tag around the fruit stem. Put the plants back to the growth room, and let the fruit grow for about one week.

Note: Fruit coloration may start to show at about 3 days post injection.

9. Examine the fruit color and the GFP signal (if necessary) using the fluorescence dissecting stereomicroscope. Take pictures or collect tissues for downstream analysis.

Note: Cut the fruit in order to observe the internal phenotypes. If necessary, the GFP fluorescence can be used as a guide for collecting tissues for downstream analysis.

\section{Data analysis}

Fruits of the $F$. vesca accession $\mathrm{YW}$ at the large green stage (15-20 days post pollination) were used for transiently overexpressing FveMYB10 (Figure 1A). We can see that the injected fruits are full of water under the receptacle skin (Figure 1B). One week later, fruit coloration has been fully developed (Figure 1C). Frequently, some area of the receptacle turns red, while the rest remains white. When the fruit is cut into two halves, tissues close to the skin turn red, while the inner part remains white. The intensity of the GFP fluorescence correlates well with the fruit coloration (data not shown). In contrast, fruits injected with only the injection buffer (negative control) stay white (Figure 1D). Anthocyanin synthesis or ripening genes are often transiently modified in cultivated strawberry. In order to illustrate that this approach is also suitable for gene knock-down, we exhibit the cultivated strawberry fruit transiently transformed with the RAP-RNAi construct (binary vector pK7WIWG2D) (Figure 1E) (Luo et al., 2018). Of note, the parts with GFP fluorescence overlap with 
the parts possessing color change, although GFP and RAP are independently driven by the $35 \mathrm{~S}$ constitutive promoter in one construct.



Figure 1. Phenotypes of the transiently transformed strawberry fruits. A. One fruit of the $F$. vesca accession $\mathrm{YW}$ at the large green stage. $\mathrm{B}$. The same fruit from $A$ that was just injected. C. One YW fruit showing red color after one week of injecting the FveMYB10-ox construct. D. The control YW fruit injected only with the injection buffer. E. One fruit of the strawberry cultivar 'Sweet Charlie' showing reduced anthocyanin accumulation after one week of injecting the RAP-RNAi construct. Right images showing the GFP signal taken by the fluorescence microscope. Scale bars $=1 \mathrm{~cm}$.

\section{$\underline{\text { Notes }}$}

1. Due to the limitation of fruit developmental stages used for injection, Agrobacterium-mediated transformation is more suitable for studying genes modulating late developmental processes, such as fruit ripening and the formation of fruit quality (coloration, formation of aroma and taste, etc.).

2. The transformation efficiency varies greatly among individual fruit, even within one fruit. We suggest using vectors carrying a visible reporter to facilitate phenotype observation and tissue collection. GFP or other fluorescent proteins are better options than the $\beta$-glucuronidase (GUS).

3. Grow the plants at about $25^{\circ} \mathrm{C}$. Lower or higher temperatures $\left(30^{\circ} \mathrm{C}\right)$ will greatly reduce the efficiency or even lead to a complete failure.

4. We succeeded in transforming a mixture of agrobacteria harboring two constructs, respectively, in a ratio of 1:1. This provides more potentials of examining the interactions between two or more genes.

5. Besides F. vesca and F. $\times$ ananassa, this approach should be applicable to other Fragaria species as well.

6. In this transient strawberry transformation, no acetosyringone is used. In the strawberry stable transformation, acetosyringone is added into the incubation buffer, and a few hours' incubation of the cell suspension is required. This optimization might be tested in the future. 


\section{Recipes}

1. Luria Broth (LB) medium

$10 \mathrm{~g} \mathrm{~L}^{-1}$ Tryptone

$5 \mathrm{~g} \mathrm{~L}^{-1}$ Yeast extract

$10 \mathrm{~g} \mathrm{~L}^{-1} \mathrm{NaCl}$

$\mathrm{pH} 7.0$

$15 \mathrm{~g} \mathrm{~L}^{-1}$ Agar (for the solid LB medium)

2. Injection buffer

$0.44 \mathrm{~g}$ MS powder

$2 \mathrm{~g}$ Sucrose

$100 \mathrm{ml} \mathrm{dd} \mathrm{H}_{2} \mathrm{O}$

pH 5.8

3. Antibiotics

$50 \mathrm{mg} \mathrm{L}^{-1} \mathrm{Gent}$

$50 \mathrm{mg} \mathrm{L}^{-1}$ Rif

$50 \mathrm{mg} \mathrm{L}^{-1}$ Spe

\section{Acknowledgments}

This work in Kang's lab was supported by the National Natural Science Foundation of China (31572098, 31772274, and 31822044). This protocol was modified from our previously published work (Luo et al., 2018).

\section{Competing interests}

All the authors declare that they have no competing interests.

\section{$\underline{\text { References }}$}

1. Hawkins, C., Caruana, J., Schiksnis, E. and Liu, Z. (2016). Genome-scale DNA variant analysis and functional validation of a SNP underlying yellow fruit color in wild strawberry. Sci Rep 6: 29017.

2. Hoffmann, T., Kalinowski, G. and Schwab, W. (2006). RNAi-induced silencing of gene expression in strawberry fruit (Fragaria $\mathrm{x}$ ananassa) by agroinfiltration: a rapid assay for gene function analysis. Plant J 48(5): 818-826.

3. Huang, D., Wang, X., Tang, Z., Yuan, Y., Xu, Y., He, J., Jiang, X., Peng, S. A., Li, L., Butelli, E., Deng, $X$. and $X u, Q$. (2018). Subfunctionalization of the Ruby2-Ruby1 gene cluster during the domestication of citrus. Nat Plants 4(11): 930-941. 
4. Jia, H. F., Chai, Y. M., Li, C. L., Lu, D., Luo, J. J., Qin, L. and Shen, Y. Y. (2011). Abscisic acid plays an important role in the regulation of strawberry fruit ripening. Plant Physiol 157(1): 188-199.

5. Li, C., Yamagishi, N., Kasajima, I. and Yoshikawa, N. (2019). Virus-induced gene silencing and virus-induced flowering in strawberry (Fragaria $\times$ ananassa) using apple latent spherical virus vectors. Horticulture research 6: 18-18.

6. Lin-Wang, K., McGhie, T. K., Wang, M., Liu, Y., Warren, B., Storey, R., Espley, R. V. and Allan, A. C. (2014). Engineering the anthocyanin regulatory complex of strawberry (Fragaria vesca). Front Plant Sci 5: 651.

7. Luo, H., Dai, C., Li, Y., Feng, J., Liu, Z. and Kang, C. (2018). Reduced Anthocyanins in Petioles codes for a GST anthocyanin transporter that is essential for the foliage and fruit coloration in strawberry. J Exp Bot 69(10): 2595-2608.

8. Spolaore, S., Trainotti, L. and Casadoro, G. (2001). A simple protocol for transient gene expression in ripe fleshy fruit mediated by Agrobacterium. $J$ Exp Bot 52(357): 845-850.

9. Tang, T., Yu, X., Yang, H., Gao, Q., Ji, H., Wang, Y., Yan, G., Peng, Y., Luo, H., Liu, K., Li, X., Ma, C., Kang, C. and Dai, C. (2018). Development and validation of an effective CRISPR/Cas9 vector for efficiently isolating positive transformants and transgene-free mutants in a wide range of plant species. Front Plant Sci 9: 1533. 